\title{
A Study of the Behavior of Cement Concrete under the Vibratory Action of a Vibrostand
}

\author{
Liu Ying ${ }^{1, a}$, Peng Peng ${ }^{1, b}$, Li Chuanping ${ }^{2, c}$ \\ 1. Research Institute of Highway, Ministry of Transport, Beijing, 100088; \\ 2. Chang'an University, Xi'an, 710064 \\ ay.liu@rioh.cn, bp.peng@rioh.cn, ${ }^{\mathrm{c}} 1115317328 @ q q . c o m$
}

Keywords: Cement Concrete, Vibratory Action, Vibrostand

Abstract: By addressing itself to the vibrational liquefaction feature of cement concrete and modeling based on the behavior of cement concrete under the vibratory action of a vibrostand, this paper comes to the differential equations of motion of element granules in concrete and, based on the model, analyzes the vibrational liquefaction mechanism of cement concrete and the effect of vibration parameters on the vibrational behavior of cement concrete to advantage guidance on cement concrete project construction.

In indoor cement concrete proportioning tests, a vibrostand has to be used to compact concrete by vibrational liquefaction, so as to increase its workability and strength. Through researches on the rheological feature of a fresh concrete mixture, we can consider it as a Bingham solid composed of a Hooke solid and a visco-plastic solid; under forced vibrations, the vibration system composed of elements, such as sand granule, and their periphery has certain stiffness $k$ and damping $c$. Mathematic modeling of the vibration system advantages researches on the behavior of cement concrete under the vibratory action of a vibrostand.

\section{Making a cement concrete vibration model}

To make the vibration model of a mixture, we have to study the exciting force of the vibrostand first. The following is a simplified schematic operational diagram of the vibrostand. Its differential equation of motion is established:

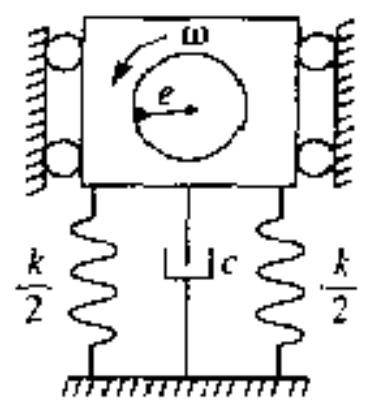

$$
\left(M-m_{0}\right) \ddot{x}+m_{0} e w^{2} \sin (w t+\varphi)+m_{0} \ddot{x}=-k_{0} x-c_{0} \ddot{x}
$$

which is simplified as:

$$
M x+c_{0} x+k_{0} x=m_{0} e w^{2} \sin (w t+\varphi)
$$

in which: $M$ - gross mass of the vibrostand; 


$$
\begin{aligned}
& \mathrm{m}_{0} \text { - eccentric mass; } \\
& e \text { - eccentricity; } \\
& \mathrm{k}_{0} \text { - total stiffness of the spring; } \\
& \mathrm{c}_{0} \text { - vibration system damping. }
\end{aligned}
$$

Thus the operation of the vibrostand can be considered as system response, with harmonic excitation of the square of the frequency, created by rotation of eccentric mass, as excitation.

And then, we have to study the travel of vibration waves in concrete. The travel of vibration waves in concrete can be considered as that of elastic waves in a semi-continuous medium. Within a unit time, the energy of vibration waves arriving at height $h$ from the vibrostand is

$$
E=C e^{-\alpha h}
$$

in which: $C$ - integration constant;

$\alpha$ - coefficient of energy loss, relevant to material properties.

The vibrations of the vibration system composed of elements in the concrete mixture at height $h$ from the vibrostand, such as sand granule, and their periphery can be considered as SDOF forced vibrations with certain stiffness; before the vibrations, elements, such as sand, remain relatively balanced under the action of dead weight, buoyancy and surrounding material. With a unit time, work done by the exciting force at $h$ is

$$
W=\frac{a}{2} A w^{2}
$$

in which $A_{h}$ is the amplitude of vibration waves arriving at $h$.

$$
E=W
$$

So

$$
A_{h}=\frac{1}{w} \sqrt{\frac{2 C}{c}} e^{-\frac{\alpha}{2} h}
$$

This is the general rule of amplitude decay when vibration waves travel in concrete. $A_{0}$ is assumed as amplitude on the surface of the vibrostand and then the following can be drawn from Eq (6)

$$
\frac{A_{h}}{A_{0}}=e^{-\frac{\alpha}{2} h}
$$

The differential equation of motion of the SDOF forced vibration system with certain stiffness, which is composed of elements in the concrete mixture at height $h$ from the vibrostand, such as sand granule, and their periphery, can be finally drawn

$$
m \ddot{x}+c \dot{x}+k x=m_{0} e w^{2} e^{-\frac{\alpha}{2} h} \sin (w t+\varphi)
$$

or

$$
\ddot{x}+2 \xi w_{n} \dot{x}+w_{n}{ }^{2} x=\frac{m_{0}}{m} e w^{2} e^{-\frac{\alpha}{2} h} \sin (w t+\varphi)
$$

The general solution to $\mathrm{Eq}(9)$ is the free vibrations of an SDOF system with viscous damping. In the case of small damping, the form of the general solution is

$$
x_{1}(t)=A e^{-\xi w_{n} t} \sin \left(w_{d}+\varphi\right)
$$

The vibration decays and disappears at last. The particular solution to it is worked out as

$$
x_{2}(t)=\frac{B_{0}}{\left.\sqrt{\left(1-r^{2}\right.}\right)^{2}+(2 \xi r)^{2}} \sin \left(w t-\tan ^{-1} \frac{w c}{K-w^{2} m}\right)
$$

in which: $B_{0}=\frac{m_{0} e w^{2} e^{-\frac{\alpha}{2} h}}{K}, r=\frac{w}{w_{n}}$. It can be known from Eq (11) that the amplitude of forced 
vibrations becomes greatest and system vibrations become most intense when $r=1$ or $w=w_{n}$.

The vibration acceleration of elements, such as gravel, can be drawn from Eq (11):

$$
\ddot{x}_{2}(t)=-\frac{m_{0} e w^{4} e^{-\frac{\alpha}{2} h}}{\left.K \sqrt{\left(1-r^{2}\right.}\right)^{2}+(2 \xi r)^{2}} \sin \left(w t-\tan ^{-1} \frac{w c}{K-w^{2} m}\right)
$$

Then the size of inertia force on them is

$$
I=m \ddot{x}_{2}(t)=-\frac{m m_{0} e w^{4} e^{-\frac{\alpha}{2} h}}{\left.K \sqrt{\left(1-r^{2}\right.}\right)^{2}+(2 \xi r)^{2}} \sin \left(w t-\tan ^{-1} \frac{w c}{K-w^{2} m}\right)
$$

The size of inertia force on element granules has an immediate effect on their vibrational behavior under the vibratory action of the vibrostand.

\section{Vibrational liquefaction mechanism of cement concrete}

Cement concrete is composed of sand, stone, water, cement and some additives. Cement paste composed of cement and water plays a lubricating role before hardening and a cementing role after hardening. Sand and pebbles represent more than $70 \%$ of the volumetric composition of concrete material, playing a filling role as aggregate. In addition, there are pores and free water that make up $1 \%$ to $5 \%$ of the volume.

Before cement concrete is vibrated, main effective stresses on elements, such as gravel, as a result of the action of gravity, cohesion and internal friction are tensile-compressive stress and shear stress which are largely concentrated at contact points between element granules. At the early stage of vibration, element granules, which are under the action of vibration waves, will be subject to the repeated action of vertical and shear stresses that vary constantly in cyclicity, size and direction. Because of different mass of element granules, their different location and difference in surface shape and arrangement status, initial stresses and live load applied between element granules and at their different positions will be different such that element granules will be subject to a remarkably varying effect under the action of vibration waves, hence a change in shear stress between element granule contact points. Element granules remain in their original positions when the shear stress does not suffice to overcome the cohesion.

The shear stress will reduce the cohesion between element granules while it is building up; when such a shear stress exceeds a certain value, it will damage the bonding strength and structural status between original element granules such that they will get out of contact. Then, the effective pressures originally transferred via granule contact points will be borne by free water in pores and gives rise to pressure waves; the water pressure transferred by pressure waves and that arising from squeeze between element granules jointly cause an increase in free water pressure and a decrease in the effective stresses between element granules, reducing the effective shearing strength of concrete. When the free water pressure builds up gradually to such an extent that it suffices to overcome mixture's surface tension and cohesive forces, such as capillary force, as well as physical absorption, mechanical meshing and adhesive forces, such as hydrogen bond, mixture's original interlock-meshing structure is damaged, the internal friction greatly decreases, the effective stresses on granules get virtually lost, and element granules stay approximately in a suspended state.

Element granules come into vibration under the action of vibration waves; some of them with particular mass even come into resonance. Free water squeezes out upwards along with release of pore water pressure; at the same time, gas remaining in concrete floats and spills upwards in the form of air bubbles, whereas element granules sink slowly, under the action of dead weight and upward water pressure, in an attempt to occupy the most stable position on the bottom layer, stacked and arranged anew. At this moment, concrete has a shearing strength that equals to zero and shows the properties of a liquid. This process is known as vibrational liquefaction. After vibrations 
stop, element granules, mutually interlocked and meshed, form a new compacted structure.

\section{Effect of vibration parameters on the vibrational behavior of cement concrete}

It's not hard to see from the so-made model that the behavior of cement concrete under the vibratory action of the vibrostand relates to factors like the vibration frequency, amplitude and vibration acceleration of the stand and material properties. Studies on the effect of these factors and reasonable selection of relevant parameters are of great significance for practical production.

Vibration frequency

To achieve the best vibratory compaction result, it's hoped that the vibration frequency of the vibrostand lies close to the inherent frequency of cement concrete mixture so that, with the phenomenon of resonance occurring, element granules have the greatest amplitude and get more energy. However, we can hardly get the inherent frequency of cement concrete mixture due to the heterogeneity of element granules plus uncertain displacement arising from vibrations. Usually, we determine the frequency of the vibration system using the size of element granules (which decides their mass). Generally speaking, a greater size corresponds to greater mass, larger inertia and lower inherent frequency; in other words, the vibration frequency will decrease as the size increases. There are a variety of granules of different size in the mixture designed according to the proportioning, and it's necessary to choose the size with the highest content or granules of average size to determine the vibration frequency. The vibration frequency of the vibrostand can be adjusted by changing the engine speed (motor type) or electric current frequency (electromagnetic type); according to practical experience, $50-100 \mathrm{~Hz}$ is normally chosen as the vibration frequency.

\section{Amplitude}

The amplitude of the vibrostand has an immediate effect on the transfer of cement concrete mixture energy; the greater the amplitude is, the more kinetic energy is transferred and the more liable to overcome the internal friction and come into displacement. Selection of the amplitude relates to mixture properties and vibration frequency. Generally speaking, mixtures with perfectly round smooth-faced uniform granules, gradation of small granules in a large quantity and high slump have good flowability and small frictional drag between element granules and are liable to displacement under the action of vibration waves; therefore a relatively small amplitude can be chosen. It's necessary to choose a greater amplitude for concrete of stiff consistency with low slump as it is not liable to flow due to great internal friction. Moreover, amplitude selection should be in concert with frequency. Lower vibration frequency usually corresponds to greater amplitude and vice versa. Coordinated selection of the amplitude and vibration frequency has a considerable effect on the travel depth of vibrations. Great amplitude and low frequency should be chosen for large-volume concrete, such as dam and pier, whereas small amplitude and high frequency are required for concrete panels, such as cement concrete pavement.

\section{Vibration acceleration}

Vibration acceleration directly decides whether or not concrete can be vibro-compacted and is critical for subsequent strength. During vibrational liquefaction, vibration acceleration remarkably affects concrete cohesion. In the process of growth of the vibration acceleration transferred into a concrete mixture, concrete viscosity will decrease, the internal friction will greatly decrease, and element granules will be liable to motion. Certainly, vibration acceleration must not increase unlimitedly as excessively great vibration acceleration may result in concrete segregation and lamination, having a considerable effect on concrete's structural strength while reducing vibrator's durability. Usually, the lower the concrete slump is, the greater the vibration acceleration is; 5-10 g is chosen as vibration acceleration, as the case may be.

\section{Vibration time}

It takes some time, but preferably not an inappropriately long while, to respond to the vibrational excitation of concrete granules with certain inertia. It's known from Eq (13) that the greater mass 
the element granules have, the greater inertia force they have and the more liable they are to overcome the internal friction, move downwards under the action of gravity and thus occupy a lower-layer position. Element granules with small mass, however, will be stacked, interlocked and arranged on the higher layer and then get segregated and laminated, which is unallowable as mentioned earlier. Moreover, the longer the vibrations last, the higher tendency and higher extent of such segregation and lamination there will be. Therefore in practice, vibrations should be stopped when a lot of mortar appears and no more air bubbles come out. At this moment, small granules fill in gaps between large granules and finer mortar fills in gaps between small granules so that the mixture becomes adequately dense. The optimum vibration time largely depends on the quality and volume of concrete mixture. The vibration time on a vibrostand is $3-5 \mathrm{~s}$ for low-plasticity concrete or 5-10 s for concrete of stiff consistency.

\section{Conclusions}

(1) Under the action of simple harmonic excitation waves, the vibrations of element granules in concrete are lagging simple harmonic ones of the same frequency at initial stage of vibrational liquefaction and their amplitude decreases exponentially with increasing travel distance (decay of excitation waves).

(2) The repeated action of vibration waves on vertical and shear stresses on element granules, which vary constantly in cyclicity, size and direction, will, at first, gradually lower effective stresses at granule contact points where stresses are concentrated, separate them, and result in great reduction of internal friction.

(3) Buildup of the pressure waves of free water in gaps makes free water squeeze out upwards and element granules are rearranged slowly under the action of dead weight and upward water pressure; meanwhile, the gas comes out upwards and, at this stage, concrete has a shearing strength that equals to zero and shows the properties of a liquid. This is vibrational liquefaction.

\section{References}

[1] Li Wanli, Yan Rongqing, Li Ziguang. A Study of the Vibration Parameters of SF-350 Cement Concrete Paver Vibrating System [J]. Journal of Changsha Communications University, 1996, 12(2): 28-32.

[2] Yang Shimin. Mechanical Properties of Aggregate Type Materials \& Vibrational Liquefaction Mechanism of Roller Compacted Concrete [J]. Concrete, 2000(8): 47-49.

[3] Wu Aixiang. Bulk Solid's Vibrational liquefaction and Compacting Action Testing \& Mechanism Discussions [J]. Journal of Central-South Institute of Mining and Metallurgy, 1991(23): 15-20.

[4] Hu Yongbiao, Chen Die, Zhao Jianlin. A Study of Vibro-compaction Acceleration Decay Coefficient in Slipform Concrete [J]. Journal of Xi' an Highway University, 1999, 19(4): 36-38. [5] Sun Nanping. Analysis of the Laitance Mechanism of Cast-in-situ Fly Ash Concrete [J]. Concrete, 2005(12): 74-112.

[6] Fu Zhi. A Study of the Vibrational Structural Viscosity of Fresh Concrete [J]. Journal of Highway and Transportation Research and Development, 1996(3): 1-9.

[7] Zhang Yimin. Mechanical Vibration [M].1. Beijing: Tsinghua University Press, 2007.

[8] He Tingji, Hu Yongbiao. Cement Concrete Pavement Construction \& Construction Machinery [M].1. Beijing: People's Communication Press, 2000: 44-67. 